as more such factors are affinity-purified, cloned and eventually synthesized in bulk. The second may be less straightforward. So far, none of these factors has been shown to be very tissue specific or to increase transcription even 100 -fold in vivo or in vitro, yet the expression of eukaryotic genes can vary from tissue to tissue over many orders of magnitude. Even the factor $\mathrm{NF}-\kappa \mathrm{B}^{6}$, which binds to the immunoglobulin $\kappa$ enhancer and seemed to be confined by $\kappa$-producing cells, now appears to be inducible in HeLa cells by phorbol ester treatment ${ }^{\text {? }}$ (although it differs in several ways from the Sp1-type factors described here).

The possibility therefore remains that almost all the trans-acting factors uncovered in the remarkable discoveries of the

NEWSANDVIEWS

past three years constitute a mechanism for the fine-tuning of transcription in a given cell, and that the availability of a particular gene for transcription is determined by a separate class of trans-acting factors working in a different and unexplored way.

1. Jones, K. A., Kadonga, J. T., Rosenfield, P. J., Kelly, T. J. \& Tjian, R. Cell 48, 79-89 (1987)

2. Briggs, M. R., Kadonga, J. T., Bell, S. P. \& Tjian, R. Science 234, 47-52 (1986)

3. Takahashi, K. et al. Nature 319, 121-126 (1986).

4. de Vries, E., van Driel, W., van den Heuvel, S. J. L. \& van der Vliet, P. C. EMBO J. 6, 161-168 (1987)

5. Prijn, G. J. M., van Driel, W. \& van der Vliet, P. C. Nature 322, 656-659 (1986)

6. Sen, R. \& Baltimore, D. Cell 46, 705-716 (1986)

. Sen, R. \& Baltimore, D. Cell 47, 921-928 (1986).

Nicholas J. Short is at the Department of Bio physics, King's College London (KQC), 26-29 Drury Lane, London WC2B $5 R L, U K$.

\title{
Drosophila genetics
}

\section{Love-song and circadian rhythm}

\section{Michael Ashburner}

Biological clocks allow organisms to synchronize their activities with the predictable cycle of day and night, giving them a sense of time, so that even in the absence of external cues an organism's behaviour changes during the day as it would under normal conditions. A population of the fruitfly Drosophila, for example, entrained to a 12-h light:12-h dark cycle, will continue to hatch just after dawn, and only then, even in constant darkness. At least six different genes are known in D.melanogaster to affect circadian rhythms. The best studied of these is the per gene, discovered by Ron Konopka when a graduate student at Caltech. New results reported by $\mathrm{Yu}$ and colleagues on page 765 of this issue' suggest a mechanism by which this gene mediates two different effects.

Mutation of per abolishes completely all circadian behaviour, not only the hatching rhythm but also the rhythm of spontaneous locomotor activity of adult flies. Other mutations of this gene can increase the period of the rhythm from 24 to $29 \mathrm{~h}$ $(p e r)$ or decrease it to $19 \mathrm{~h}\left(\right.$ per $\left.^{\prime}\right)$. Mutations of this gene also have remarkable effects on a rhythm with a very different periodicity - the oscillation in the interval between the bursts of sound that characterize the love-song male Drosophila sing to potential mates. This interval varies around a mean of $34 \mathrm{~ms}$ in D. melanogaster with a period of about $55 \mathrm{~s}$ in the wild type. In per males this period is $80 \mathrm{~s}$, in per it is $40 \mathrm{~s}$ and in per there is no rhythmicity in the variation in interpulse interval.

The temporal characteristics of the male love-song are highly species-specific. For example, in the closely related species D.simulans the mean interval between pulses of song is $49 \mathrm{~ms}$, fluctuating with a period of $40 \mathrm{~s}$. Females are excited by a song characteristic of their own species, not that of another. More strikingly, hybrid females prefer the (simulated) song of hybrid males ${ }^{2}$. This raises two questions. The first is the role of the per gene in the female; does it perhaps encode an oscillator against which incoming song is measured? If so, then this would neatly explain the genetic coupling between the male's stimulus and the female's response. The second is how the product of one gene can affect a species-constant circadian rhythm and a rhythm that is not only variable between species but whose very variation is concerned with species isolation.

Whether per encodes a component of the fundamental oscillator or a component of the black box that lies between the oscillator and the behaviour is not yet known. Perhaps molecular studies of per and its gene products will help ${ }^{3,4}$. These studies are certainly providing fascinating insights into the genetic control of behaviour. As reported by $\mathrm{Yu}$ et al. in this issue' the effects of per on the very short period rhythms of the love-song and on circadian rhythms can be experimentally distinguished. Judging from its DNA sequence, per encodes a very large protein of 1,218 amino acids. These include a run of alternating glycine and threonine residues. The length of the Gly-Thr run varies between different wild-type per alleles: in some stocks it is 17 Gly-Thr repeats, in others 20 or 23 . It is not known whether or not these different alleles are functionally different. We would certainly like to know whether males with these different alleles show the same periodicity characteristics of their love-songs. By some nifty cutting and splicing Yu et al. ${ }^{1}$ constructed a per gene that lacks the DNA encoding the Gly-Thr repeat region and used it to transform per $^{\rho}$ flies. The surprising result is that this mutant construct can rescue the circadian rhythm phenotype to normality but that the flies have a very short love-song rhythm. The per ${ }^{\circ}$ flies transformed with a normal per gene have a love-song periodicity of about $60 \mathrm{~s}$; when transformed by the Gly-Thr deletion they have a periodicity of about $40 \mathrm{~s}$. In principle, this result gives a mechanism by means of which the periodicity of the lovesong can evolve without affecting the control of circadian rhythms.

The love-song of per flies transformed with the deleted gene shows the same periodicity, about $40 \mathrm{~s}$, as that of $\mathrm{per}^{s}$, known to be a single amino-acid substitution about 100 residues amino-terminal to the Gly-Thr region ${ }^{5,6}$. The periodicity of circadian rhythms in per transformants is negatively correlated with amount of per messenger RNA ${ }^{6}$, aithough periodicities shorter than $24 \mathrm{~h}$ have not yet been achieved by over-expression. It is possible to reconcile these observations in a single model if we assume that the circadian oscillation depends on the absolute amount of per gene product and that the song rhythm depends on variations in per protein concentration. As a first approximation it is easy to see how the amount of per protein could oscillate around a mean if, for example, the protein is unstable and blocked the translation of its own messenger RNA. Then a change in its stability (for example, by a change in the number of Gly-Thr repeats) will change the periodicity of oscillation of protein concentration. Such speculation is, however, probably premature. Although we know that the per protein is a proteoglycan-like molecule $e^{3.7}$, there are also clear indications that the per gene encodes at least three different but related proteins ${ }^{4}$ that may be involved in different aspects of its function. Complementary DNAs encoding two of these proteins can rescue the circadian phenotype of $\mathrm{per}^{\circ}$ flies. Moreover, there remains a large black box between per and its proteins and the behavioural phenotype and there is plenty of room for other genes and their products to interact with those of per. That having been said, we are but on the threshold of an exciting analysis of the genetic and molecular basis of a very fundamental behavioural pattern.

1. Yu, Q. et al. Nature 326, 765-769 (1987).

2. Kyrlacou, C. P. \& Hall, J.C. Science 232, 494-497 (1986).

Jackson, F.R., Bargiello, T.A., Yun, S.-H. \& Young, Jackson, F.R., Bargiello, T.A.,

4. Citri, Y. et al. Nature 326, 42-47 (1987)

5. Yu, Q. et al. Proc. natn. Acad. Sci. U.S.A. 84, 784-788 (1987)

6. Baylies, M.K., Bargiello, T.A., Jackson, F.R. \& Young, M.W. Nature 326, 390-392 (1987).

7. Reddy, P. Jacquier, A.C. Abovich, N., Petersen, G. \& Rosbash. M. Cell 46, 53-61 (1986).

Michael Ashburner is at the Department of Genetics, University of Cambridge, Downing Street, Cambridge CB2 $3 E H, U K$. 